\title{
Effects of Bladder Function by Early Tamsulosin Treatment in a Spinal Cord Injury Rat Model
}

\author{
Kang Keun Lee, $\mathrm{MD}^{1}$, Moon Young Lee, $\mathrm{MD}^{2}$, Dong Yeop Han, $\mathrm{MD}^{3}$, \\ Hee Jong Jung, $\mathrm{MD}^{3}$, Min Cheol Joo, $\mathrm{MD}^{1}$
}

${ }^{1}$ Department of Rehabilitation Medicine and Institute of Wonkwang Medical Science, Wonkwang University School of Medicine, Iksan; Departments of ${ }^{2}$ Physiology and ${ }^{3}$ Urology, Wonkwang University School of Medicine, Iksan, Korea

Objective To investigate the effects of early tamsulosin treatment on changes in bladder characteristics after a spinal cord injury.

Methods We divided 45 rats into three groups: the control (CON) group, the spinal cord injury (SCI) group, and the SCI+tamsulosin treatment (SCI+TAM) group. Spinal cord transection was performed in the SCI and SCI+TAM groups. Tamsulosin was injected for 7 days in the SCI+TAM group. Intravesical and intra-abdominal catheters were implanted before cord injury. Basal pressure (BP), maximal vesical pressure (MVP), micturition volume (MV), and voiding interval time (VIT) were measured at 7 days after SCI. The bladder was then removed and used for an in vitro organ bath study and Western blot analysis. The percentage changes in contractility from baseline after acetylcholine alone, pretreatment with a muscarinic 2 (M2) receptor blocker (AQ-RA741), and pretreatment with a M3 receptor blocker (4-DAMP) were compared among the groups. Western blot analyses were performed to determine expression levels of pERK1/2 and rho-kinase.

Results In cystometry, MVP, BP, MV, and VIT showed changes in the SCI and SCI+TAM groups versus the CON group $(p<0.05)$. In the organ bath study, acetylcholine-induced contractility in the three groups differed significantly $(\mathrm{p}<0.05)$. Additionally, acetylcholine-induced contractility with 4-DAMP pretreatment was reduced significantly in the SCI+TAM group versus the SCI group. In Western blotting, pERK1/2 expression was stronger $(\mathrm{p}<0.05)$ and rho-kinase expression was weaker in the SCI+TAM group than the SCI group $(\mathrm{p}<0.05)$.

Conclusion These results suggest that the bladder contraction due to acetylcholine after SCI can be decreased by tamsulosin in the acute stage and this involves changes in pERK1/2 and rho-kinase.

Keywords Spinal cord injuries, Tamsulosin, Neurogenic urinary bladder

Received February 7, 2014; Accepted March 25, 2014

Corresponding author: Min Cheol Joo

Department of Rehabilitation Medicine and Institute of Wonkwang Medical Science, 895 Muwang-ro, Iksan 570-974, Korea

Tel: +82-63-859-1621, Fax: +82-63-843-1385, E-mail: mcjoo68@wku.ac.kr

(c) This is an open-access article distributed under the terms of the Creative Commons Attribution Non-Commercial License (http://creativecommons. org/licenses/by-nc/3.0) which permits unrestricted noncommercial use, distribution, and reproduction in any medium, provided the original work is properly cited.

Copyright $\odot 2014$ by Korean Academy of Rehabilitation Medicine

\section{INTRODUCTION}

A spinal cord injury in the upper sacrum can block sensory information from the micturition center to the brain and descending motor information from the brain to the micturition center. When the brain is unable to control the micturition center in patients who have suffered a spinal cord injury, the micturition center works independently, resulting in non-voluntary bladder contraction, 
also known as an overactive bladder [1].

The storage and excretion of urine in the bladder is controlled by interactions among three nerves in the lower urinary tract (the parasympathetic, sympathetic, and somatic nerves) and the central nervous system. Contraction of the bladder is dominated by the parasympathetic nervous system, with acetylcholine as the mediator of the muscarinic receptors in the bladder. The storage of urine and relaxation of the bladder is stimulated mainly by the sympathetic nervous system, mediated by adrenergic receptors.

In an animal model, it has been reported that the activation of $\mathrm{C}$-fibers and changes in the muscarinic receptor in the bladder may cause non-inhibitory and non-voluntary bladder contractions after a spinal cord injury [2]. Changes in muscarinic receptors in the bladder after a spinal cord injury are known generally to be caused by changes in the sensitivity of the receptors and an increased density of the M2 receptor due to a subtype change, from M3 to M2 [3]. Adrenergic receptors include $\alpha$ - and $\beta$-receptors. Adrenergic $\alpha$-receptors are usually found in the urethral muscle and the vesical trigone, but are rare in the detrusor. The symptoms of overactive bladder are caused by changes in the sensitivity of the $\alpha_{1 D}$-adrenergic receptor in the detrusor or direct instability of the detrusor.

An overactive bladder causes micturition disorders, affecting urinary frequency and incontinence. The primary drugs for treatment are anti-cholinergic agents; $\alpha$-blockers are also used. $\alpha$-Blockers, including tamsulosin, are known to be effective in benign prostate hyperplasia and to decrease urinary function after a spinal injury [4].

Additional effects of tamsulosin on the smooth muscle in the bladder, peripheral ganglion, and spinal cord have been reported. Price [5] reported that tamsulosin not only improved micturition, by working in the lower urinary tract, but also blocked the $\alpha_{1 \mathrm{D}}$-adrenergic receptor in the detrusor during the bladder filling period, to help store urine and decrease the sensitivity of the bladder. The same report added that tamsulosin decreased the secretion of acetylcholine in the peripheral cholinergic nerve in the bladder or the peripheral ganglion, eventually decreasing non-voluntary contraction of the bladder.

Given this background, the sensitivity of the bladder in a spinal injury model was investigated using an in vitro organ bath study and in vivo awake internal bladder pressure measurements. Moreover, changes in the activities of pERK1/2, rho-kinase, and acetylcholine in the bladder were investigated after the injury, and the effects of different subtypes of muscarinic receptors on bladder contraction and of early tamsulosin treatment on the changes in an overactive bladder caused by spinal injury were investigated.

\section{MATERIALS AND METHODS}

\section{Experimental animals and spinal cord injury}

Animal experiments were performed in accordance to the institutional guidelines provided by the Committee on Animal Research at Wonkwang University. Efforts were made to minimize both animal suffering and the number of animals.

We purchased 45 female Sprague Dawley rats, weighing 250-270 g, from Samtaco (BioKorea, Osan, Korea). They were divided into three groups: a group with no surgical intervention (CON group, $\mathrm{n}=12$ ), a spinal injury-induced group (SCI group, $\mathrm{n}=12$ ), and a $\mathrm{SCI}+$ tamsulosin treatment group (SCI+TAM group, $\mathrm{n}=21$ ). All surgical procedures were performed by the same investigator. All rats received $300 \mathrm{mg} / \mathrm{kg}$ chloral hydrate to induce anesthesia. Complete spinal cord transection was performed at the T10 vertebral level. After the completion of all surgical procedures, the surgical area was sterilized. Gentamicin $(100 \mathrm{mg} / \mathrm{kg}$ ) was injected intramuscularly for 5 days to prevent wound infection.

\section{Insertion of a catheter into the bladder}

For the polyethylene catheter insertion, the rats were anesthetized intraperitoneally, and an incision was made to locate the catheter in the bladder dome 5 days before the spinal damage. The catheter exited through the subcutaneous cavity on the back of the rats. Cystostomy was performed in all animals, and a spinal injury was introduced 5 days after the animals were well stabilized. The animals in the SCI+TAM group were injected with 0.1 $\mathrm{mg} / \mathrm{kg}$ of tamsulosin intraperitoneally, twice per day for 7 consecutive days. Their internal bladder pressure was measured while they were conscious 7 days after the spinal injury. Then, they were sacrificed to investigate bladder function, and an organ bath study was conducted. The catheter for the measurement of the internal bladder pressure was inserted 5 days before the spinal injury, as mentioned above [6]. 


\section{Drugs}

The acetylcholine M2 receptor antagonist 11-[[4-[4-(diethylamino)butyl]-1-piperidinyl] acetyl]-5,11-dihydro6H-pyrido[2,3-b][1,4] benzodiazepin-6-one (AQ-RA741) and the M3 receptor antagonist 1,1-dimethyl-4-diphenylacetoxypiperidinium iodide (4-DAMP) were purchased from Sigma (South Croydon, Australia). For tamsulosin (Astellas Pharma Inc., Tokyo, Japan), $0.1 \mathrm{mg} / \mathrm{kg}$ was injected intraperitoneally every 12 hours for 7 days in the SCI+TAM group.

\section{Organ bath study}

All rats were sacrificed by cervical dislocation 7 days after the spinal injury. The abdomen was opened to extract the bladder. The specimens were sectioned vertically, and part of the detrusor was placed in an organ bath filled with oxygenated Krebs solution $\left(95 \% \mathrm{O}_{2}, 5 \% \mathrm{CO}_{2}\right)$ at $37^{\circ} \mathrm{C}$.

The detrusor was cut into $1-\mathrm{cm}$ sections. One edge of the section was fixed at the base of the organ bath, and the other edge was connected to a force transducer (FT03; Grass Technologies, Warwick, RI, USA). The preload tension was adjusted to $1 \mathrm{~g}$. The tissue samples were stabilized for at least 60 minutes to achieve consistent contraction, and the degree of contraction was recorded regularly. Various concentrations of acetylcholine $\left(10^{-9}\right.$ to $10^{-4} \mathrm{M}$ ) were administered continuously, and the contractions were recorded according to the concentration of acetylcholine. Other tissue samples were pre-treated with M2 and M3 receptor antagonists, and the area under the curve (AUC) immediately before the administration of acetylcholine (ACh) and until 5 minutes after the administration was obtained by applying a higher concentration of acetylcholine to compare the contractility of the samples.

Contractility $(\%)=($ AUC after ACh administration/AUC before ACh administration) $\times 100$

All data were recorded using a PowerLab data acquisition system (AD Instruments, Sydney, Australia).

\section{Western blot analysis}

The expression levels of phosphorylated extracellular signal-regulated kinases $1 / 2$ (pERK 1/2) and rho-kinase were measured using Western blot analysis. Bladder tis- sues were washed with phosphate-buffered saline, and approximately $5 \mathrm{~g}$ tissue was added to lysis buffer (20 mM Tris-acetate, $10 \mathrm{mM} \mathrm{NaCl}, 0.1 \mathrm{mM}$ EDTA, $1 \mu \mathrm{g} / \mathrm{mL}$ aprotinin, $\mathrm{pH}$ 7.4) and homogenized. The samples were then centrifuged (12,000 rpm, 30 minutes), and the cytosolic fraction was obtained. The protein concentration was estimated using the Bradford method, with bovine serum albumin (BSA) as the standard. The protein concentration of all samples was adjusted so as to apply the same amount of protein. Sample buffer was added to the samples, the mixture was boiled at $100^{\circ} \mathrm{C}$ for 5 minutes, and proteins were separated by electrophoresis. The gels were then stained with Coomassie brilliant blue R-250 for 1 hour. Then, the gels were destained with $10 \%$ acetic acid and $10 \%$ methanol, and the protein bands were observed. The proteins were transferred to $0.45 \mu \mathrm{m}$ polyvinylidene difluoride (PVDF) membranes (Millipore Co., Bedford, MA, USA) using a protein transfer apparatus. To prevent non-specific binding of the primary antibody, the PVDF membranes were incubated with blocking buffer (3\% BSA in Tris-buffered saline [TBS], pH 7.6) for 1 hour. The membranes were incubated overnight at $4^{\circ} \mathrm{C}$ with primary antibodies (pERK1/2, 1:500) and washed three times with $0.05 \%$ Tween 20 -TBS (TBST). Next, the membranes were incubated for 1 hour with a conjugated goat anti-rabbit IgG secondary antibody (Santa Cruz Biotechnology Inc., Santa Cruz, CA, USA; 1:5,000). The membranes were washed with alkaline phosphatase (0.1 M Tris, $0.1 \mathrm{M} \mathrm{NaCl}, 0.01 \mathrm{MgCl}_{2}$ ) after being washed twice with TBST. The color reaction used nitro-blue tetrazolium and 5-bromo-4-chloro-3'-indolyl-phosphate, p-toluidine salt (BCIP).

\section{Awake cystometry}

The bladder pressure was measured in the animal house while the animals were conscious. The catheter inside the bladder was connected to a two-way valve, and a pressure transducer (Research-grade Blood Pressure Transducer; Harvard Apparatus, Holliston, MA, USA) and a micro-injection pump (PHD22/2000 pump; Harvard Apparatus) were connected to it using a T-tube 7 days after the spinal injury. In total, $6.0 \mathrm{~mL}$ of normal saline at room temperature was injected into the bladder at 0.06 $\mathrm{mL} / \mathrm{min}$, and the change in internal bladder pressure was recorded in a log book. The mean voided volume in a fluid collector connected to the force displacement trans- 
ducer was recorded.

The base pressure, maximum bladder pressure, voided volume, and frequency of urination were recorded constantly using the MP150 data acquisition system (BIOPAC Systems, Goleta, CA, USA) and the AcqKnowledge software ver. 3.8.1 (BIOPAC Systems).

\section{Data analysis}

Data were analyzed using the SPSS software ver. 11.0 (SPSS Inc., Chicago, IL, USA). Differences in the contractility of tissues treated with different compounds were determined for the CON, SCI, and SCI+TAM groups using an independent $\mathrm{t}$-test. The $\mathrm{p}$-values $<0.05$ were considered to indicate statistical significance.

\section{RESULTS}

\section{Effects of acetylcholine on voluntary contractions}

In the organ bath study, the contractility in both the SCI group and the SCI+TAM group increased more significantly with the increase in acetylcholine concentration than in the CON group. However, the increase in contractility in the SCI+TAM group was significantly smaller than in the SCI group $(\mathrm{p}<0.05)$ (Fig. 1).

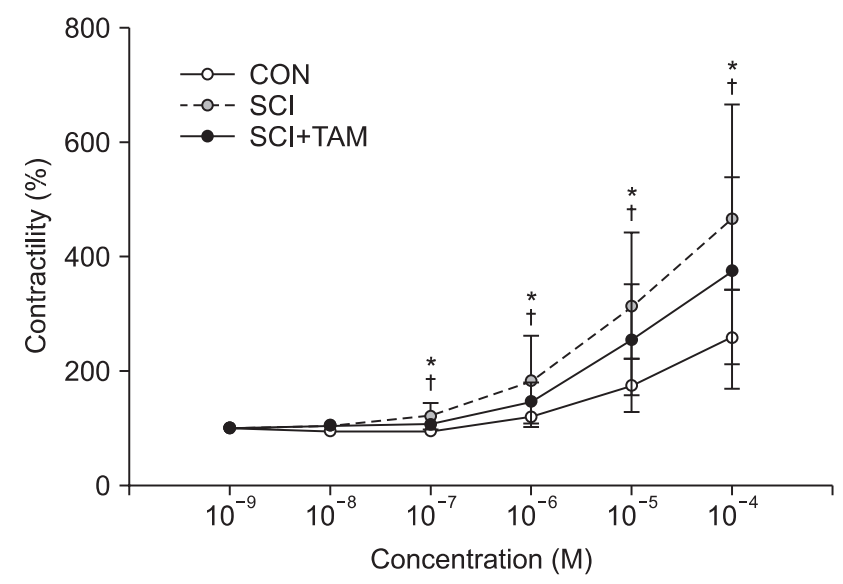

Fig. 1. Percentage changes in contractility for the CON, SCI, and SCI+TAM groups. The intergroup difference in the percentage changes in contractility was significant. The values are expressed as mean \pm standard deviation. CON, control; SCI, spinal injury-induced; TAM, tamsulosin. *Denotes significant difference between the CON group and the others $(\mathrm{p}<0.05) .{ }^{\dagger}$ Denotes significant difference between the SCI group and the SCI+TAM group $(\mathrm{p}<0.05)$.
Response to acetylcholine after pre-treatment with AORA741 and 4-DAMP

The contractility in response to acetylcholine after pretreatment with AQ-RA741 showed no significant differ-

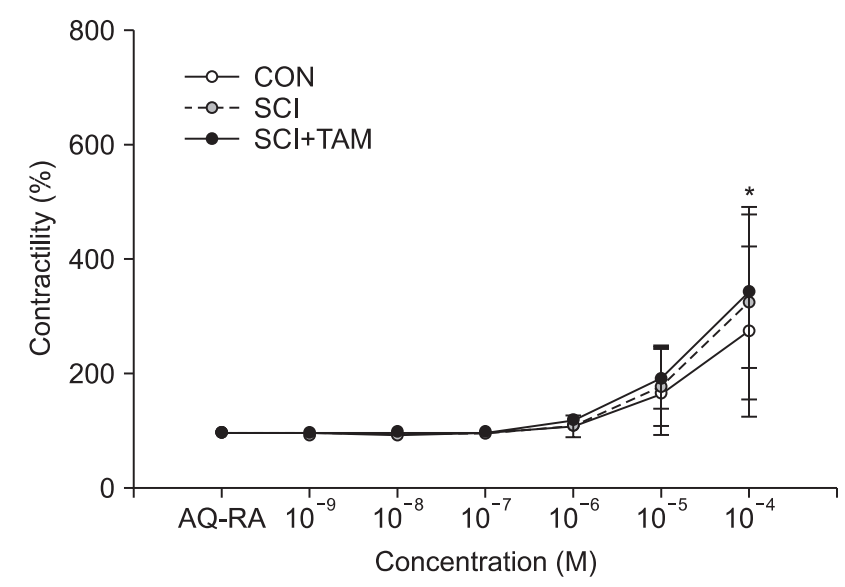

Fig. 2. Percentage changes in contractility after pretreatment with $\mathrm{M} 2$ receptor antagonist (AQ-RA) for the CON, SCI, and SCI+TAM groups. The intergroup difference in the percentage changes in contractility was not significant. The values are expressed as mean \pm standard deviation. CON, control; SCI, spinal injury-induced; TAM, tamsulosin. *Denotes significant difference between the CON group and the others $(\mathrm{p}<0.05)$.

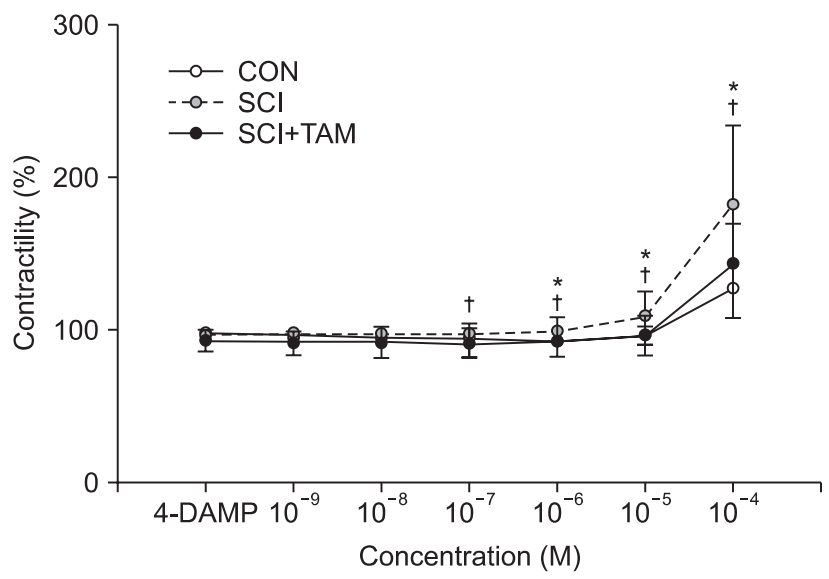

Fig. 3. Percentage changes in contractility after pretreatment with M3 receptor antagonist (4-DAMP) for the CON, SCI, and SCI+TAM groups. The intergroup difference in the percentage changes in contractility was significant. The values are expressed as mean \pm standard deviation. CON, control; SCI, spinal injury-induced; TAM, tamsulosin. *Denotes significant difference between the CON group and the others $(\mathrm{p}<0.05) .{ }^{\dagger}$ Denotes significant difference between the SCI group and the SCI+TAM group $(\mathrm{p}<0.05)$. 
ence in the three concentration groups, except in the $10^{-4}$ $M$ group ( $>>0.05$ ) (Fig. 2). The contractility in response to acetylcholine after pre-treatment with 4-DAMP increased more significantly in both the SCI group and the SCI+TAM group than in the CON group, but the SCI+TAM group showed significantly lower results than the SCI group $(\mathrm{p}<0.05)$ (Fig. 3).

\section{Awake cystometry results}

The urination point was associated with an increase in internal bladder pressure in the CON group, whereas neither the SCI group nor the SCI+TAM group showed any correlation between the increase in internal bladder pressure and urination points (Fig. 4). The internal blad- der pressure data are shown in Fig. 4 and Table 1. The differences in the base pressures and the maximum bladder pressures of the SCI group and the SCI+TAM group versus the CON group were $38.5 \%$ and $62.5 \%$, and $63.7 \%$ and $47.9 \%$, respectively, showing significant decreases $(\mathrm{p}<0.05)$ (Fig. 5). The differences in the voided volumes and urination frequencies in the SCI group and the SCI+TAM group versus the CON group were $36.8 \%$ and $38.6 \%$, and $54.2 \%$ and $39.2 \%$, respectively, both showing significant decreases $(\mathrm{p}<0.05)$ (Fig. 6). However, there was no significant difference in the base pressures, maximum bladder pressures, voided volumes, or urination frequencies of the SCI and SCI+TAM groups.
CON

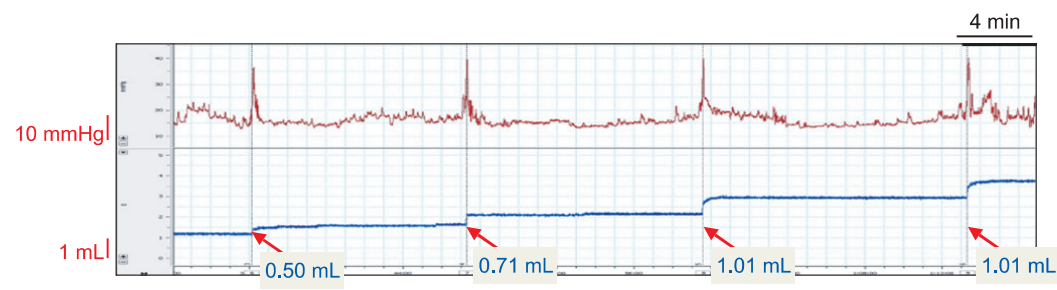

$\mathrm{SCl}$

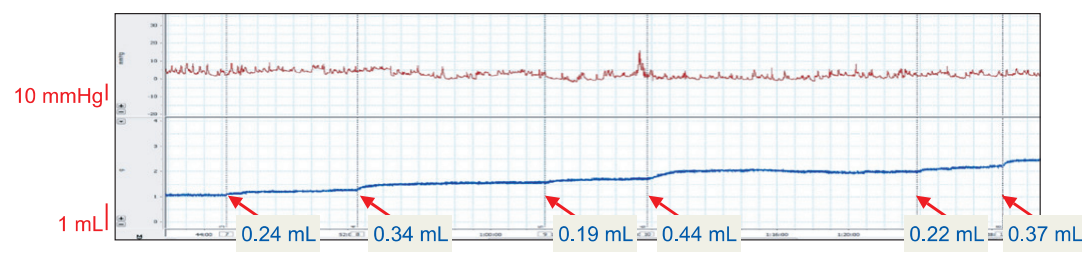

SCI+TAM

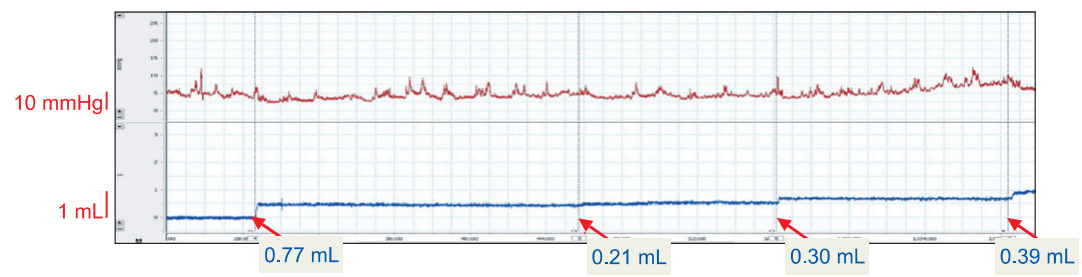

Fig. 4. Results of awake cystometry. In the control, increases in the pressure of the bladder and urinary voiding time shown a matching pattern. However, the SCI and SCI+TAM groups did not match. The SCI group has a more frequent voiding pattern. CON, control; SCI, spinal injury-induced; TAM, tamsulosin.

Table 1. BP, MVP, MV, and VIT of the CON, SCI, and SCI+TAM groups

\begin{tabular}{lrcc}
\hline & CON & SCI & SCI+TAM \\
\hline BP (mmHg) & $13.88 \pm 3.44(100)$ & $5.35 \pm 8.75(38.5)^{*}$ & $8.68 \pm 4.06(62.5)^{*}$ \\
MVP (mmHg) & $40.17 \pm 9.55(100)$ & $25.59 \pm 15.15(63.7)^{*}$ & $19.25 \pm 10.24(47.9)^{*}$ \\
MV (mL) & $1.14 \pm 0.40(100)$ & $0.42 \pm 0.22(36.8)^{*}$ & $0.44 \pm 0.19(38.6)^{*}$ \\
VIT (sec) & $992.93 \pm 368.19(100)$ & $538.10 \pm 507.87(54.2)^{*}$ & $389.15 \pm 237.21(39.2)^{*}$ \\
\hline
\end{tabular}

Values are presented as mean \pm standard deviation (\%).

There were significant difference between the CON group and the others. However, there was no significant difference between the SCI group and the SCI+TAM group.

CON, control; SCI, spinal injury-induced; TAM, tamsulosin; BP, basal pressure; MVP, maximal vesical pressure; MV, micturition volume; VIT, voiding interval time.

*Denotes significant difference between the CON group and the others $(\mathrm{p}<0.05)$. 
(A)

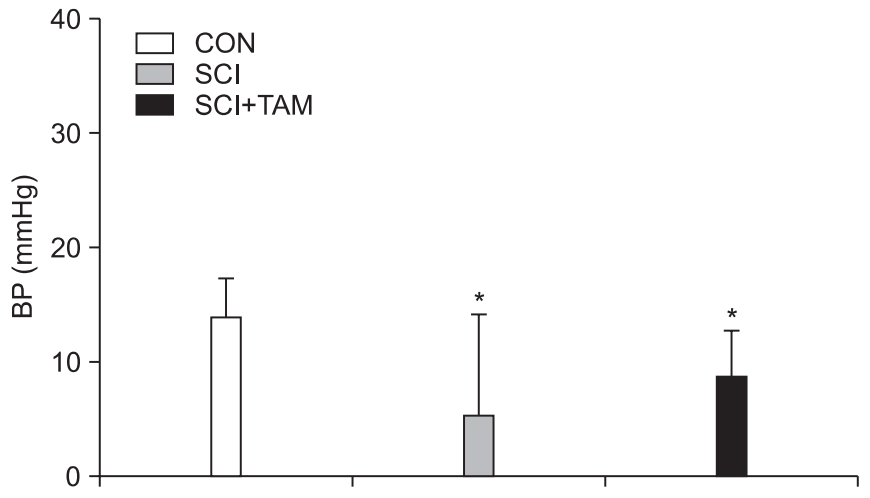

(B)

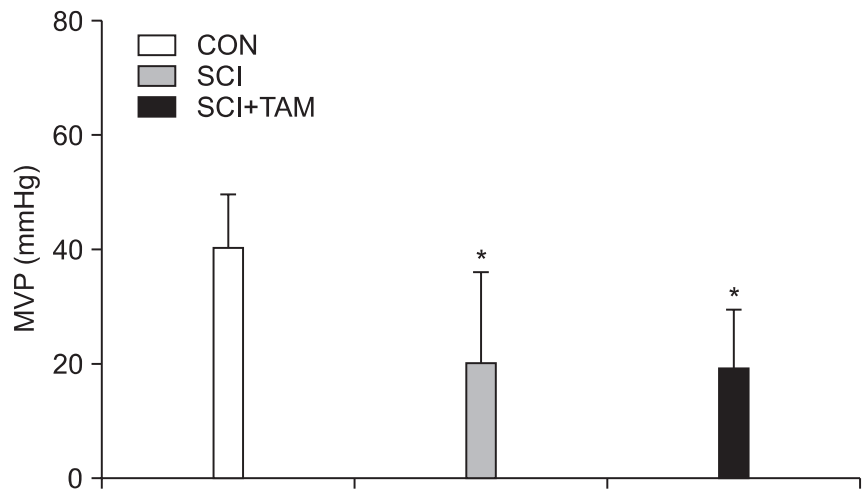

Fig. 5. Basal pressure (BP, A) and maximal vesical pressure (MVP, B) of the CON, SCI, and SCI+TAM groups; there are significant difference between the CON group and the others. However, there was no significant difference between the SCI and SCI+TAM groups. CON, control; SCI, spinal injury-induced; TAM, tamsulosin. *Denotes significant difference between the CON group and the others $(\mathrm{p}<0.05)$.

(A)

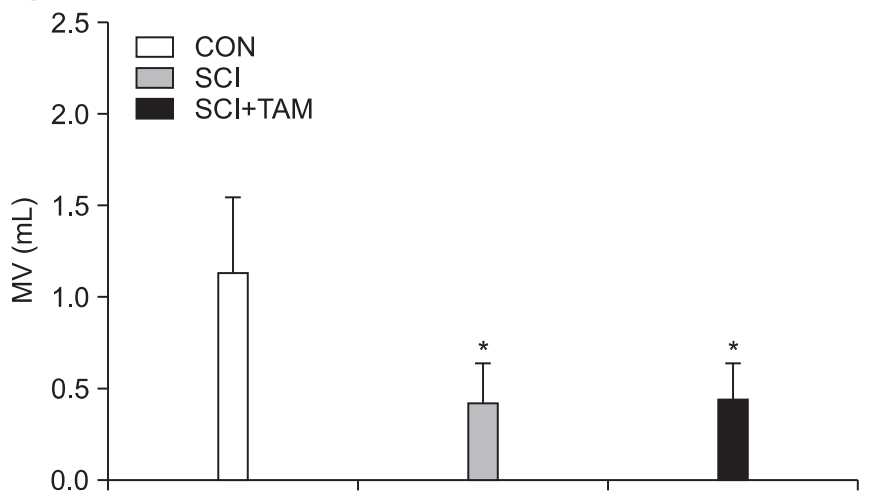

(B)

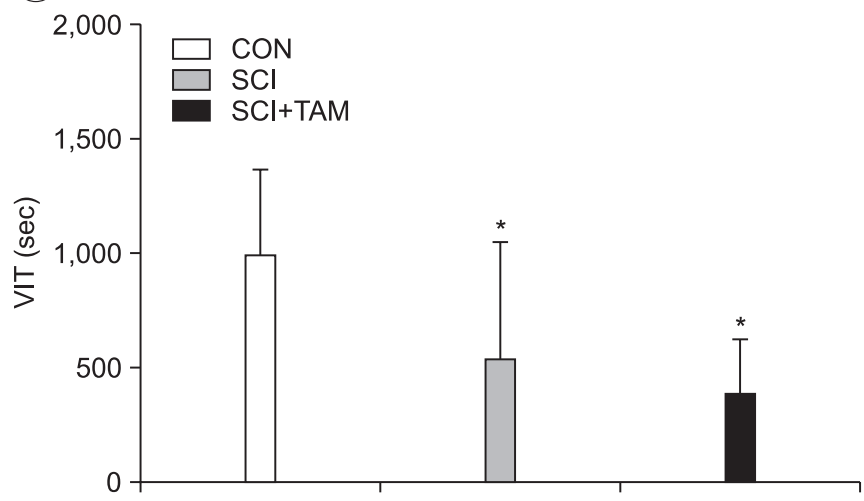

Fig. 6. Micturition volume (MV, A) and voiding interval time (VIT, B) of the CON, SCI, and SCI+TAM groups; there are significant difference between the CON group and the others. However, there was no significant difference between the SCI and the SCI+TAM groups. CON, control; SCI, spinal injury-induced; TAM, tamsulosin. *Denotes significant difference between the CON group and the others $(\mathrm{p}<0.05)$.

\section{Western blot}

The Western blot showed no difference in ERK1/2 expression in the three groups. However, the expression of $\mathrm{pERK} 1 / 2$ decreased significantly $(\mathrm{p}<0.05)$, and that of rho-kinase increased significantly $(\mathrm{p}<0.05)$, in the SCI group than in the CON group or the SCI+TAM group (Fig. 7).

\section{DISCUSSION}

In normal bladder physiology, contraction of the muscles of the bladder can be inhibited by an $\alpha_{1}$-adrenergic receptor antagonist [7]. This explains the effect of tera- zosin, one of the $\alpha_{1}$-adrenergic receptor antagonists that improves compliance of the bladder and increases the bladder volume [8]. Recent studies have also reported the presence and functions of $\alpha_{1 D}$-adrenergic receptors in the detrusor [9], and animal studies have shown that $\alpha_{1}$-adrenergic receptors are associated with storage symptoms [10]. A significant change in the $\alpha_{1}$-adrenergic receptor subtypes was observed in animals with bladder obstruction. In the CON group, $70 \%$ of the animals had the $\alpha_{1 \mathrm{~A}}$-adrenergic receptor subtype, $16 \%, \alpha_{1 \mathrm{D}}$, and $14 \%$, $\alpha_{1 \mathrm{~B}}$. In the bladder obstruction group, $91 \%$ of the animals had the $\alpha_{1 D}$-adrenergic receptor subtype, and 5\%, $\alpha_{1 \mathrm{~A}}$. These results were taken to mean that the increased ex- 
(A)

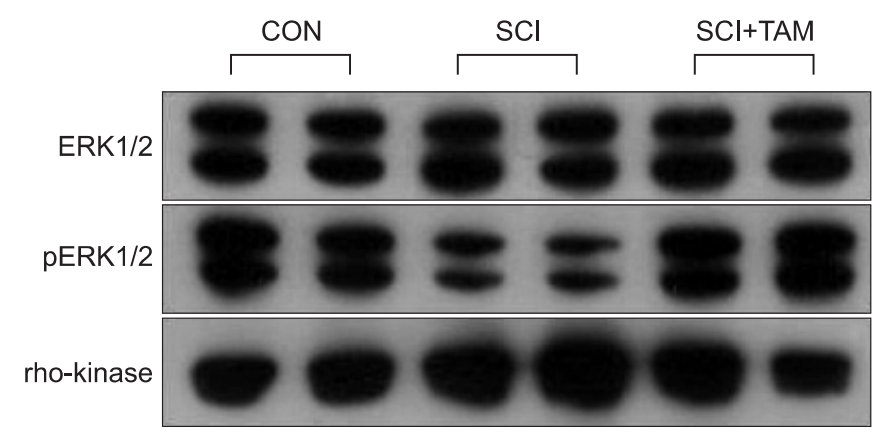

(C)

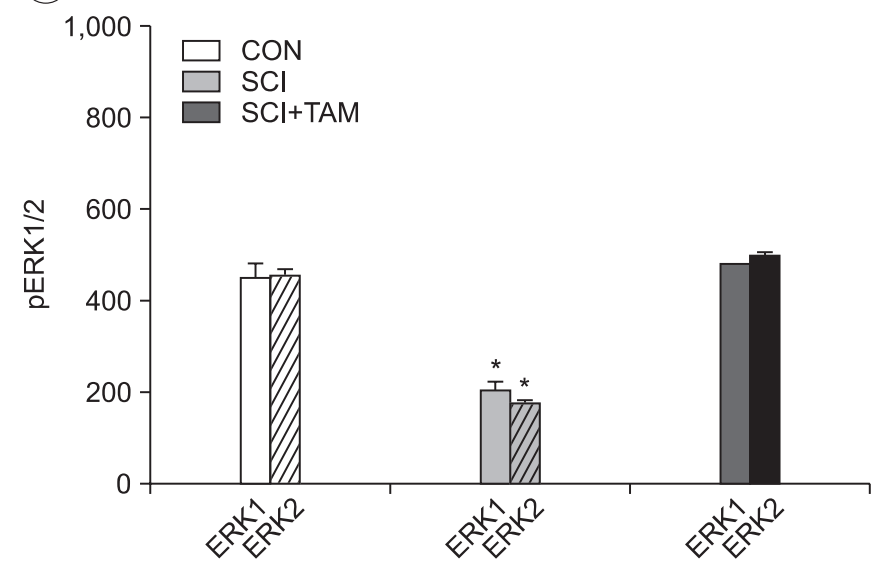

(B)

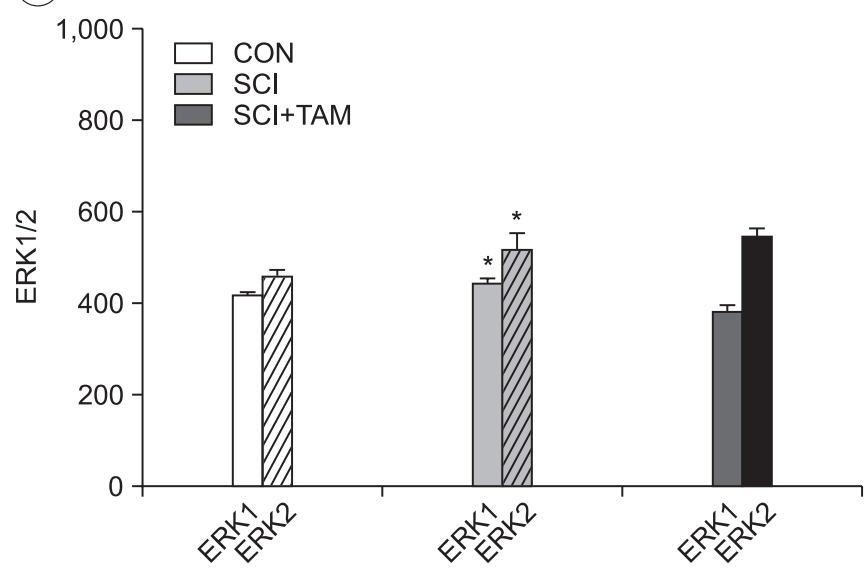

(D)

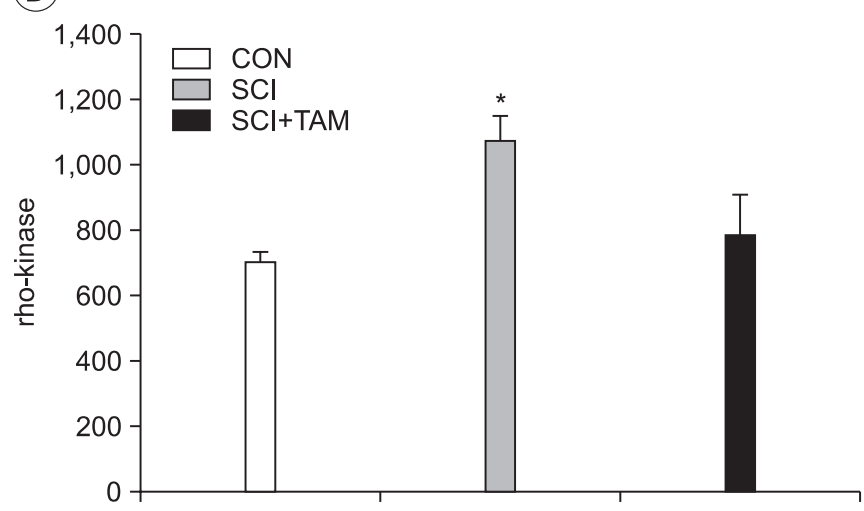

Fig. 7. Results of Western blot analysis for extracellular signal-regulated kinases $1 / 2$ (ERK1/2, B), phosphorylated ERK1/2 (pERK1/2, C), and rho-kinase (D) in the bladder. (A) shows representative cases for each protein. Note the prominent expression of pERK1/2 and lower expression of rho-kinase in the SCI+TAM group than in the SCI group. (B), (C), and (D) showed the optical density analysis for ERK1/2, pERK1/2, and rho-kinase. CON, control; SCI, spinal injury-induced; TAM, tamsulosin. *Denotes significant difference between the SCI group and the others $(\mathrm{p}<0.05)$.

pression of the $\alpha_{1 D}$-adrenergic receptor subtype then affected urination behavior, and they partly demonstrated a relationship between the symptoms and the $\alpha_{1 D}$ receptor subtype [11].

Recent studies have provided more objective evidence of the effects of tamsulosin on the bladder [12]. Tamsulosin is a potent and selective $\alpha_{1}$-adrenergic receptor antagonist that is known to be more effective at the $\alpha_{1 \mathrm{~A}}$ and $\alpha_{1 \mathrm{D}}$ receptors than the $\alpha_{1 \mathrm{~B}}$ receptor [13]. Tamsulosin also improves bladder storage and urination in patients with spinal injuries in the upper sacrum, and decreases the symptoms associated with abnormal reflexes of the automatic nerve system [14].

The micturition reflex of rats is blocked at the acute stage of a spinal cord injury, but the reflex restarts a few weeks after the injury, causing an overactive bladder [15]. Contraction of the bladder is dependent on parasympathetic stimulation of muscarinic receptors, found in the detrusor [16]. There are five muscarinic receptor subtypes (M1-M5), classified based on molecular biology and pharmacodynamics [17]. A study on competitive binding confirmed that most species have M2 and M3 receptors in their bladders [18]. The frequency of M2 is highest, but the M3 receptor contributes most to contraction of the detrusor [19].

In rats, the bladder becomes more sensitive to acetylcholine, a cholinergic transmitter, after a spinal cord injury. Increased intensity of all muscarinic receptors and a shift from M3 to M2 receptors in rats with a spinal cord injury has been reported, causing an overactive bladder 
[3]. Another study reported an increase in M2 receptors in the bladder of rats with diabetes [20].

An in vivo experiment on functional responses with various stimuli using an organ bath showed that the function of the detrusor originates from the action of the smooth muscles that are blocked in the bladder. Also, the blocked detrusor showed hypersensitivity to the muscarinic antagonists [21] and cholinergic agents in human neuropathic bladder [22].

In this study, the response to acetylcholine in vitro showed a statistically significant difference in the SCI and SCI+TAM groups versus the CON group from the lowest concentration of $10^{-7} \mathrm{M}$, and the bladder was shown to be hypersensitive to the cholinergic agents after the spinal cord injury. Also, there was a significant difference between the SCI group and the SCI+TAM group, attributed to reduced hypersensitivity of the bladder due to the early treatment with tamsulosin. There was no significant difference in the responses to acetylcholine in the three groups when pre-treated with AQ-RA741, to block M2 receptors, but there was a significant difference in the responses to acetylcholine in the CON group and the SCI group and in the SCI group and the SCI+TAM group when they were pre-treated with 4-DAMP, to block M3 receptors. These results were considered to have been because M3 receptors mediate normal bladder contraction, whereas M2 receptors are involved in bladder disorders after a spinal cord injury [3].

ERK1/2 are among the intracellular mediators, and mitogen-activated protein kinase (MAPK) and the activation of muscarinic receptors, particularly M2 receptors, are known to be involved in the formation of pERK1/2 [23]. Rho-kinase was first reported in 1996 by Amano et al. [24] and is known to be involved in smooth muscle contraction by directly phosphorylating the light chain of myosin, without increasing the intercellular $\mathrm{Ca}^{2+}$ concentration [25].

There was no significant difference in the expression of ERK1/2 in the three groups. However, the SCI group showed significantly lower expression of pERK1/2, the active form of ERK1/2, and higher expression of rhokinase, than the CON and SCI+TAM groups. The higher expression of rho-kinase in the SCI group was attributable to the injury, based on a previous study [26] that reported increased expression of RhoA mRNA 1 week after spinal injury. Continuous activation of Rho causes rho-kinase dependent myosin light chain phosphoryla- tion, increasing the contractility of the bladder. Based on a study [27] reporting that activation of Rho was involved in the MAPK pathway and showed the opposite action, of inhibiting ERK1/2 or pERK1/2, rho-kinase activation was considered to be related to decreased pERK1/2, but this needs to be confirmed. There was a significant difference in pERK1/2 and rho-kinase expression between the SCI group and the SCI+TAM group. This result was consistent with the in vitro results in this study, and early tamsulosin treatment was considered to have partially affected the contraction of the smooth muscles in the bladder after the spinal cord injury. To determine the exact mechanism, further studies on various MAPK pathways are required.

We also used an awake urodynamic test to evaluate bladder function. This awake test has the advantages of not requiring anesthesia and maintaining a normal physiological status during the test. Conventional urodynamic tests in animals use anesthetics, which affect the smooth muscles in the bladder and the central nervous system. As such, normal physiological conditions are not maintained during the test, and the results of the tests have limitations. In the awake urodynamic test used here, the SCI group and the SCI+TAM group showed significantly lower base pressures, maximum bladder pressures, and urination frequencies and volumes than the CON group. These results were attributed to the spinal injury but differed from the typical pattern of an overactive bladder in the in vitro organ bath study or the Western blot results, leaving questions as to the use of the awake urodynamic test as an evaluation tool for an overactive bladder model. Jin et al. [28] pointed out that the bladder of rats is thin, fragile, and easily torn. An awake dynamic test of a rat model with interstitial cystitis showed that the abnormally strong and thick wall of the bladder limited the physical motor range of the bladder during urination. As such, increased frequency of urination due to the increased internal bladder pressure has been reported as one of the characteristics of an overactive bladder, rather than increased internal bladder pressure. The typical relationship between pressure and volume in an overactive bladder-increased base pressure and the maximum internal bladder pressure-was not observed in this study during the awake urodynamic test. This was likely attributable to the physical characteristics of the rat bladder, as noted in the previous study. In this study, the rats were sacrificed at 7 days after the spinal cord injury. A previous 
study [29] reported that a rat model where the spinal segment was sectioned showed no response for 2 weeks and then slowly showed hypersensitivity. As such, the possibility of its full non-recovery from the shock on the 7th day could not be ruled out to explain the non-response in the test. Future studies should confirm the recovery from the spinal injury shock through an electrophysiological study at 2 weeks after the injury. The urodynamic test results of the in vivo study did not show a significant difference between the SCI+TAM group and the SCI group. This result may have been due to the insufficiency of the $0.1 \mathrm{mg} / \mathrm{kg}$ dose of tamsulosin in vivo, although this dose did affect contraction of the bladder in vitro. Thus, further studies are required to investigate the effective dose of tamsulosin.

Considering the limitations of this study, further studies on the recovery point in rat models of spinal cord injury from a spinal injury shock, confirmation of the introduction of overactive bladder, the tamsulosin dose and its administration point and evaluation point, and appropriate methodology for the urodynamic test should be performed.

In conclusion, there were significant differences in the responses to acetylcholine in the smooth muscles of the bladder in the CON group and the SCI group/SCI+TAM group. The response to acetylcholine after pre-treatment with AQ-RA741 showed no significant difference in the three groups, whereas the response to acetylcholine after pre-treatment with 4-DAMP did show a significant difference between the CON group and the SCI group and between the SCI group and the SCI+TAM group. These results were attributed to the increased density of the M2 receptor or the increased sensitivity of the muscarinic receptors in the bladder after the spinal cord injury. Early treatment with tamsulosin was shown to affect the contractility of the bladder after a spinal cord injury.

\section{CONFLICT OF INTEREST}

No potential conflict of interest relevant to this article was reported.

\section{ACKNOWLEDGMENTS}

This paper was supported by Wonkwang University in 2013.

\section{REFERENCES}

1. Kakizaki H, Koyanagi T. Current view and status of the treatment of lower urinary tract symptoms and neurogenic lower urinary tract dysfunction. BJU Int 2000;85 Suppl 2:25-30.

2. de Groat WC. A neurologic basis for the overactive bladder. Urology 1997;50(6A Suppl):36-56.

3. Braverman AS, Ruggieri MR Sr. Hypertrophy changes the muscarinic receptor subtype mediating bladder contraction from M3 toward M2. Am J Physiol Regul Integr Comp Physiol 2003;285:R701-8.

4. Nickel JC. The use of alphal-adrenoceptor antagonists in lower urinary tract symptoms: beyond benign prostatic hyperplasia. Urology 2003;62(3 Suppl 1):3441.

5. Price D. Potential mechanisms of action of superselective alpha(1)-adrenoceptor antagonists. Eur Urol 2001;40 Suppl 4:5-11.

6. Lee T, Andersson KE, Streng T, Hedlund P. Simultaneous registration of intraabdominal and intravesical pressures during cystometry in conscious rats: effects of bladder outlet obstruction and intravesical PGE2. Neurourol Urodyn 2008;27:88-95.

7. Anderson KE. Pharmacology of lower urinary tract smooth muscles and penile erectile tissues. Pharmacol Rev 1993;45:253-308.

8. Swierzewski SJ 3rd, Gormley EA, Belville WD, Sweetser PM, Wan J, McGuire EJ. The effect of terazosin on bladder function in the spinal cord injured patient. J Urol 1994;151:951-4.

9. Perlberg S, Caine M. Adrenergic response of bladder muscle in prostatic obstruction: its relation to detrusor instability. Urology 1982;20:524-7.

10. Hampel C, Dolber PC, Smith MP, Savic SL, Th roff JW, Thor KB, et al. Modulation of bladder alphal-adrenergic receptor subtype expression by bladder outlet obstruction. J Urol 2002;167:1513-21.

11. Docherty JR. Subtypes of functional alpha1-adrenoceptor. Cell Mol Life Sci 2010;67:405-17.

12. Robinson D, Cardozo L, Terpstra G, Bolodeoku J; Tamsulosin Study Group. A randomized double-blind placebo-controlled multicentre study to explore the efficacy and safety of tamsulosin and tolterodine in women with overactive bladder syndrome. BJU Int 2007;100:840-5. 
13. Chapple CR. Alpha-adrenergic blocking drugs in bladder outflow obstruction: what potential has alpha 1-adrenoceptor selectivity? Br J Urol 1995;76 Suppl 1:47-55.

14. Abrams P, Amarenco G, Bakke A, Buczynski A, CastroDiaz D, Harrison S, et al. Tamsulosin: efficacy and safety in patients with neurogenic lower urinary tract dysfunction due to suprasacral spinal cord injury. J Urol 2003;170(4 Pt 1):1242-51.

15. de Groat WC, Kawatani M, Hisamitsu T, Cheng CL, Ma $\mathrm{CP}$, Thor $\mathrm{K}$, et al. Mechanisms underlying the recovery of urinary bladder function following spinal cord injury. J Auton Nerv Syst 1990;30 Suppl:S71-7.

16. Caulfield MP. Muscarinic receptors: characterization, coupling and function. Pharmacol Ther 1993;58:31979.

17. Caulfield MP, Birdsall NJ. International Union of Pharmacology. XVII. Classification of muscarinic acetylcholine receptors. Pharmacol Rev 1998;50:279-90.

18. Hegde SS, Eglen RM. Muscarinic receptor subtypes modulating smooth muscle contractility in the urinary bladder. Life Sci 1999;64:419-28.

19. Chess-Williams R. Muscarinic receptors of the urinary bladder: detrusor, urothelial and prejunctional. Auton Autacoid Pharmacol 2002;22:133-45.

20. Tong YC, Chin WT, Cheng JT. Alterations in urinary bladder M2-muscarinic receptor protein and mRNA in 2-week streptozotocin-induced diabetic rats. Neurosci Lett 1999;277:173-6.

21. Sibley GN. The physiological response of the detrusor muscle to experimental bladder outflow obstruction in the pig. Br J Urol 1987;60:332-6.

22. German K, Bedwani J, Davies J, Brading AF, Stephenson TP. Physiological and morphometric studies into the pathophysiology of detrusor hyperreflexia in neuropathic patients. J Urol 1995;153:1678-83.

23. Cook AK, Carty M, Singer CA, Yamboliev IA, Gerthoffer WT. Coupling of M(2) muscarinic receptors to ERK MAP kinases and caldesmon phosphorylation in colonic smooth muscle. Am J Physiol Gastrointest Liver Physiol 2000;278:G429-37.

24. Amano M, Ito M, Kimura K, Fukata Y, Chihara K, Nakano T, et al. Phosphorylation and activation of myosin by Rho-associated kinase (Rho-kinase). J Biol Chem 1996;271:20246-9.

25. Kureishi Y, Kobayashi S, Amano M, Kimura K, Kanaide H, Nakano T, et al. Rho-associated kinase directly induces smooth muscle contraction through myosin light chain phosphorylation. J Biol Chem 1997;272:12257-60.

26. Sung JK, Miao L, Calvert JW, Huang L, Louis Harkey $\mathrm{H}$, Zhang JH. A possible role of RhoA/Rho-kinase in experimental spinal cord injury in rat. Brain Res 2003;959:29-38.

27. Kakiashvili E, Speight P, Waheed F, Seth R, Lodyga M, Tanimura S, et al. GEF-H1 mediates tumor necrosis factor-alpha-induced Rho activation and myosin phosphorylation: role in the regulation of tubular paracellular permeability. J Biol Chem 2009;284: 11454-66.

28. Jin LH, Shin HY, Kwon YH, Park CS, Yoon SM, Lee T. Urodynamic findings in an awake chemical cystitis rat model observed by simultaneous registrations of intravesical and intraabdominal pressures. Int Neurourol J 2010;14:54-60.

29. Ditunno JF, Little JW, Tessler A, Burns AS. Spinal shock revisited: a four-phase model. Spinal Cord 2004;42:383-95. 